\title{
Modificações no periodonto de ratos diabéticos após a movimentação ortodôntica
}

\author{
Luis Alberto Sabino Vila Real*, Adilson Luiz Ramos**, Jacqueline Nelisis Zanoni***
}

\begin{abstract}
Resumo
Objetivos: o objetivo deste trabalho foi avaliar as modificações do ligamento periodontal de incisivos de ratos diabéticos submetidos a forças ortodônticas. Métodos: vinte ratos machos Wistar (Rattus norvegicus) com 105 dias de idade foram empregados. Os ratos foram divididos em quatro grupos: $\mathrm{C}$ - animais normoglicêmicos não submetidos à movimentação dentária; $\mathrm{CAO}$ - animais normoglicêmicos submetidos à movimentação dentária; D - animais diabéticos não submetidos à movimentação dentária; $\mathrm{DAO}$ - animais diabéticos submetidos à movimentação dentária. Os animais permaneceram com o dispositivo de movimentação dentária por 5 dias. Foram avaliados o número de vasos sangüíneos e a espessura do ligamento periodontal nos terços cervical, médio e apical dos cortes histológicos. Resultados e Conclusões: no lado de tensão, a movimentação dentária nos animais do grupo CAO resultou em um ligamento periodontal mais espesso $(17,64 \%$ no terço apical, $39,28 \%$ no terço médio e $51,35 \%$ na região cervical), quando comparado ao grupo $\mathrm{C}$ ( $\mathrm{p}<0,05$ para os terços médio e cervical). No grupo DAO, o aumento da espessura do ligamento periodontal foi 50,55\% (terço apical), 48,14\% (terço médio) e 50\% (terço cervical) maior que nos animais do grupo D $(\mathrm{p}<0,05)$. O número de vasos sanguíneos encontrados no ligamento periodontal não apresentou diferenças estatisticamente significantes quando todos os grupos foram comparados ( $p>0,05)$. Ainda no lado de tensão, foram observadas lacunas de reabsorção nos animais dos grupos CAO, D e DAO. O lado de pressão não foi examinado nesta fase do estudo.
\end{abstract}

Palavras-chave: Movimentação dentária. Diabetes melito. Ligamento periodontal.

\section{INTRODUÇÃO}

O diabetes melito é uma patologia clínica heterogênea que se caracteriza por anormalidades endócrino-metabólicas que alteram a homeostase. As anormalidades endócrinas têm como elemento fundamental uma deficiência insulínica absoluta ou relativa, que se manifesta por uma deficiente função secretora de insulina pelo pâncreas e/ou por uma ação deficiente da insulina nos tecidos-alvo ${ }^{15}$. As anormalidades metabólicas envolvem importantes transtornos do metabolismo de carboidratos, lipídios e proteínas do organismo humano. Os sintomas mais comuns são a poliúria, polidpisia e polifagia e uma maior suscetibilidade a infecções, retinopatias, nefropatias, doenças cardiovasculares, neuropatias, osteopenia

* Graduado em Odontologia pela Universidade Estadual de Maringá - UEM.

** Doutor em Ortodontia pela Universidade Estadual Paulista Júlio Mesquita Filho-UNESP. Professor de Ortodontia do Departamento de Odontologia - UEM. Morfofisiológicas - UEM. 
e à doença periodontal ${ }^{6,12}$. Outras manifestações que podem ser encontradas são diminuições de células polimorfonucleares e da função leucocitária, metabolismo anormal do colágeno e maior tempo para a cicatrização de feridas, além de alterações no metabolismo de proteínas, que podem ser responsáveis por maior dificuldade de respostas curativas em pacientes diabéticos ${ }^{14}$. Pacientes diabéticos que apresentam seus níveis glicêmicos controlados e que tenham uma boa higiene bucal não necessariamente apresentarão manifestações bucais ${ }^{5}$.

A movimentação ortodôntica em pacientes com diabetes melito é um assunto pouco pesquisado e, diante de uma procura cada vez maior de pacientes adultos pelo tratamento ortodôntico, se faz necessária uma melhor investigação. Holtgrave et al. ${ }^{9}$ relataram que em ratos diabéticos, em conseqüência da movimentação dentária, há um retardo na regeneração óssea, enfraquecimento do ligamento periodontal e microangiopatias em áreas gengivais. Bensch et al. ${ }^{2}$ relataram que pacientes diabéticos controlados não estão contra-indicados ao tratamento ortodôntico, mas uma atenção especial deve ser dada em relação ao controle do diabetes e à doença periodontal.

A movimentação dentária induzida envolve uma série de alterações bioquímicas, que culminam com a reabsorção no tecido ósseo no lado de pressão e aposição no lado de tensão. O osso é um tecido dinâmico que sofre alterações mesmo após o final do crescimento do esqueleto, compreendendo o processo de remodelação, ou seja, um equilíbrio entre a aposição e a reabsorção óssea. A remodelação ocorre a todo momento, constituindo-se num fundamental método fisiológico de equilíbrio iônico de cálcio e fosfato. Também ocorre em função da necessidade de adaptação às forças mecânicas a que todo o esqueleto é submetido diariamente, inclusive a força gravitacional. As forças ortodônticas desencadeiam um processo remodelativo, culminando com a movimentação dentária. O processo de reabsorção ocorre constantemente e é realizado através de pequenos conjuntos de células chamados de unidades multicelulares básicas (BMU's) - que são compostas por osteoclastos, osteoblastos e macrófagos ${ }^{8}$.

Em decorrência de uma maior freqüência de pacientes diabéticos nos consultórios odontológicos à procura da correção dentária, nos motivamos a executar o presente trabalho, com o objetivo de estudar o periodonto de incisivos superiores de ratos diabéticos submetidos à movimentação dentária.

\section{MATERIAL E MÉTODOS \\ Procedimento com os animais}

Vinte ratos machos Wistar (Rattus norvegicus), pesando aproximadamente 300g, com 105 dias de idade, foram empregados. Para indução do diabetes melito, os ratos permaneceram por 14 horas em jejum e depois foi injetada - por via intravenosa - estreptozootocina ( $35 \mathrm{mg} / \mathrm{Kg}$, Sigma, USA) dissolvida em tampão citrato $10 \mathrm{mM}$ e $\mathrm{pH} 4,5$. A injeção com estreptozootocina resultou na síndrome diabética, com poliúria, polifagia e polidpsia, sendo a glicemia ${ }^{3}$ de cada animal avaliada no quinto dia após a indução do diabetes, obtendo-se o valor médio de $463 \pm 22,7 \mathrm{mg} / \mathrm{dL}$ para todos os animais diabéticos. Os ratos foram divididos em quatro grupos: $\mathrm{C}$ - animais normoglicêmicos não submetidos à movimentação dentária; $\mathrm{CAO}$ - animais normoglicêmicos submetidos à movimentação dentária; D - animais diabéticos não submetidos à movimentação dentária; DAO - animais diabéticos submetidos à movimentação dentária. Todos os animais foram pesados no início e no final do experimento.

Um dia após a indução do diabetes melito, foi realizada a instalação do dispositivo ortodôntico nos animais dos grupos CAO e DAO. Para a instalação do dispositivo ortodôntico, os ratos foram anestesiados com tiopental $(40 \mathrm{mg} / \mathrm{Kg}$ de peso corpóreo). O dispositivo constituiu-se de uma mola confeccionada com fio de aço inox de $0,35 \mathrm{~mm}$ aplicando uma força de $20 \mathrm{~g}^{1}$. A mola foi 
inserida nos incisivos centrais superiores através de um orifício feito com uma ponta carbide $1 / 2$ (KG-Soresen Ind. Com. Ltda - Brasil). O orifício foi confeccionado pela face vestibular na região do terço médio da coroa. A mola foi colocada pela face vestibular, atravessando toda a coroa dentária, sendo estabilizada por meio de uma dobra no fio na face palatina. Os dispositivos não foram reativados até a morte dos animais (Fig. 1).

Após a instalação do aparelho, os animais foram transferidos para caixas de polipropileno e receberam água e alimento ad libitum.

Os ratos foram mortos após 5 dias da instalação do aparelho, com uma injeção letal de tiopental. Antes das maxilas serem removidas, com auxílio de compasso de ponta seca e régua milimetrada, foi mensurada a distância entre as faces mesiais dos dois incisivos. As maxilas foram, então, dissecadas e removidas, sendo fixadas com formol $10 \%$ por 24 horas. Após a fixação, as molas foram removidas e as peças descalcificadas em ácido nítrico $5 \%$ durante 4 dias. A seguir, as peças foram submetidas à rotina histológica. Foram realizados cortes seriados de $5 \mu \mathrm{m}$ de espessura, no sentido longitudinal, e corados com hematoxilina e eosina.

\section{Espessura do ligamento periodontal}

A espessura do ligamento periodontal em cada animal foi analisada em seis cortes histológicos nos terços cervicais, médio e apical. Em cada região, foi realizado um total de 30 mensurações.
As mensurações foram realizadas com auxílio de régua micrometrada de Zeiss acoplada à ocular de um microscópio óptico Olympus CX31 (Olympus, Tokio Japão), utilizando a objetiva de 4X.

\section{Quantificação do número de vasos sangüíneos}

A quantificação foi realizada - nos terços cervicais, médio e apical - utilizando-se cortes semi-seriados, sendo três cortes desprezados a cada corte quantificado. Foram utilizados 5 cortes histológicos para cada animal.

\section{Tratamento estatístico}

Os dados foram analisados por meio de análise de variância (ANOVA) e o teste de Tukey foi empregado como pós-teste, para comparação de médias. As análises foram realizadas com o software Prisma 3.0 (GraphPad Software, Inc., EUA). Os resultados foram expressos como média \pm erro padrão $(\mathrm{M} \pm \mathrm{EP})$, sendo $(\mathrm{n})$ = número de ratos empregados. O nível de significância adotado foi de 5\%.

\section{RESULTADOS}

A injeção de estreptozootocina provocou, nos animais do grupo D e DAO, a síndrome diabética, constatada por severa hiperglicemia (grupo $\mathrm{D}=543,8 \pm 15,68 \mathrm{mg} / \mathrm{dL}$ e grupo DAO $384 \pm$ $29,72 \mathrm{mg} / \mathrm{dL}$ ) e também intensa polifagia, polidpsia e poliúria. Os animais do grupo $\mathrm{C}$ não tiveram variação de peso durante os 5 dias de experimento.
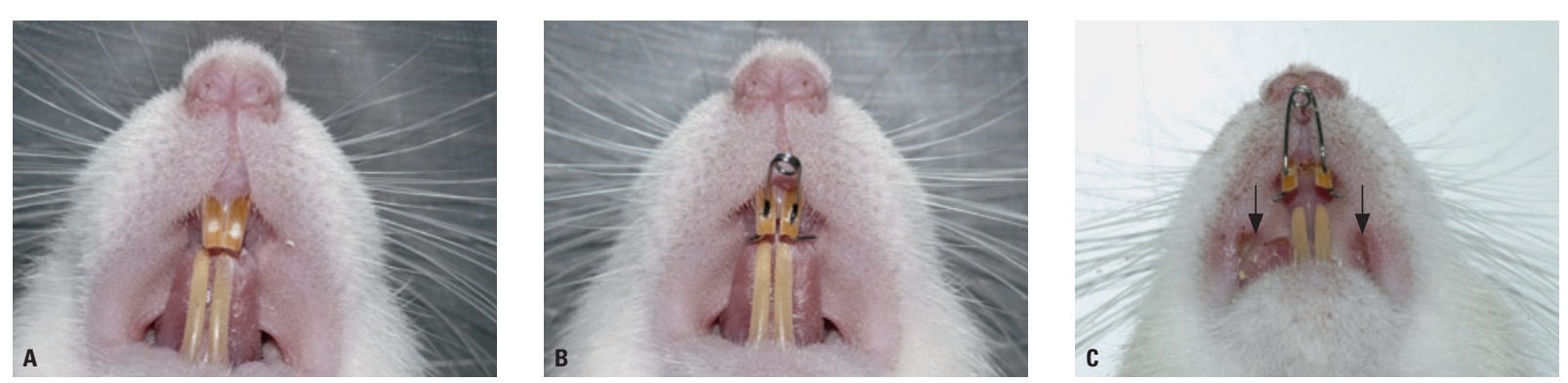

FIGURA 1 - Fotografias de ratos mostrando: A) dentes incisivos superiores antes da instalação do dispositivo ortodôntico, B) dentes incisivos superiores no primeiro dia da instalação do dispositivo ortodôntico, C) dentes incisivos superiores no quinto dia da instalação do dispositivo ortodôntico (setas = lesões semelhantes a aftas). 
Por outro lado, os animais do grupo CAO emagreceram $5 \%$ nesse período $(p>0,05)$. Os grupos $D$ e DAO apresentaram perda de peso corporal de $10,5 \%$ e $13 \%$, respectivamente, quando comparados ao grupo $\mathrm{C}(\mathrm{p}<0,05)$ (Gráf. 1).

As distâncias interincisivos observadas nos animais dos grupos $\mathrm{C}$ e D não se alteraram. No grupo CAO, a distância interincisivos foi de $1,42 \pm$ $0,14 \mathrm{~mm}$ e no grupo DAO foi de $1,48 \pm 0,16 \mathrm{~mm}$ - ao final do experimento ( $\mathrm{p}>0,05)$. A partir do segundo dia da instalação do dispositivo ortodôntico de movimentação dentária, foram observadas lesões de tecido mole - na região da comissura labial - semelhantes a aftas, nos animais dos grupos $\mathrm{CAO}$ e DAO (Fig. 1). Dois animais do grupo DAO apresentaram, no dia de sua morte, um dente com aspecto de anquilose, pois um incisivo estava mais irrompido que o outro.

A espessura do ligamento periodontal nos terços apical e médio aumentou na proporção de $36,4 \%$ e $17,64 \%$, respectivamente, nos animais do grupo D quando comparados aos do grupo C ( $p>0,05)$. A movimentação dentária nos animais normoglicêmicos (grupo CAO) resultou em ligamento periodontal mais espesso $(17,64 \%$ no terço

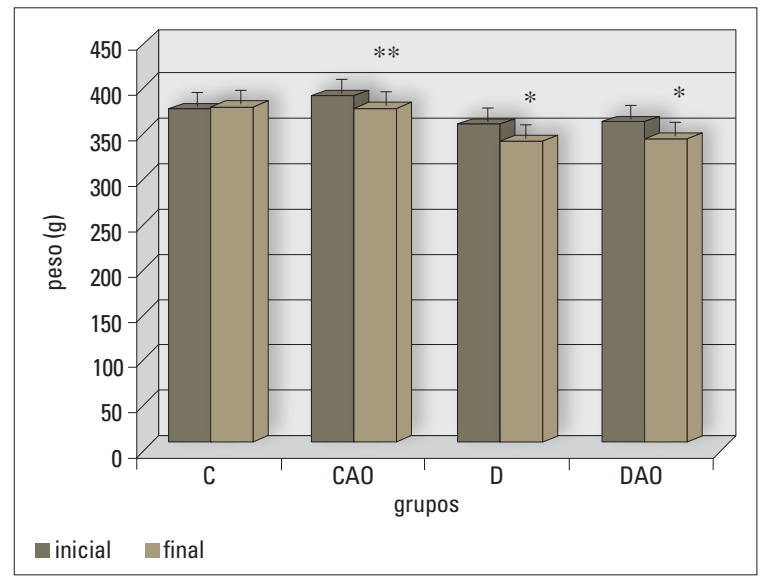

GRÁFICO 1 - Peso inicial e final (g) dos animais pertencentes aos grupos: C animais normoglicêmicos não submetidos a forças ortodônticas; CAO - animais normoglicêmicos submetidos a forças ortodônticas; $D$ - animais diabéticos não submetidos a forças ortodônticas; DAO - animais diabéticos submetidos a forças ortodônticas ( $\mathrm{n}=5$ ratos por grupo; ${ }^{*} \mathrm{p}<0,05$ quando comparado ao grupo C; ${ }^{* *} p<0,05$ quando comparado ao grupo $D$ e $p<0,01$ quando comparado ao grupo DAO). apical, 39,28\% no terço médio e 51,35\% na região cervical) quando comparados aos do grupo $\mathrm{C}(\mathrm{p}<$ 0,05 para os terços médio e cervical). O ligamento periodontal do grupo DAO foi 50,55\% (terço apical), 48,14\% (terço médio) e 50\% (terço cervical) mais espesso que o verificado nos animais do grupo D ( $\mathrm{p}<0,05)$ (Gráf. 2, Fig. 2). O ligamento periodontal dos animais diabéticos (grupos $\mathrm{D}$ e $\mathrm{DAO}$ ) apresentou um aspecto hemorrágico com extravasamento de hemácias.

Nos animais dos grupos CAO, D e DAO, o número de vasos sanguíneos encontrados no terço cervical foi $44,4 \%$, 52\% e 52,63\%, respectivamente, superior ao observado no grupo $C(p>0,05)$ (Gráf. 3).

A parede óssea no lado de tensão dos animais normoglicêmicos (grupo C) não apresentou nenhuma alteração. Por outro lado, os animais dos grupos C, D e DAO apresentaram grande quantidade de lacunas de reabsorção ao longo de toda a parede óssea (Fig. 2). Sendo que, no grupo DAO e $D$, as lacunas de reabsorção eram claramente mais pronunciadas que no grupo CAO, quando se avaliou todo o comprimento da parede óssea ao redor do dente.

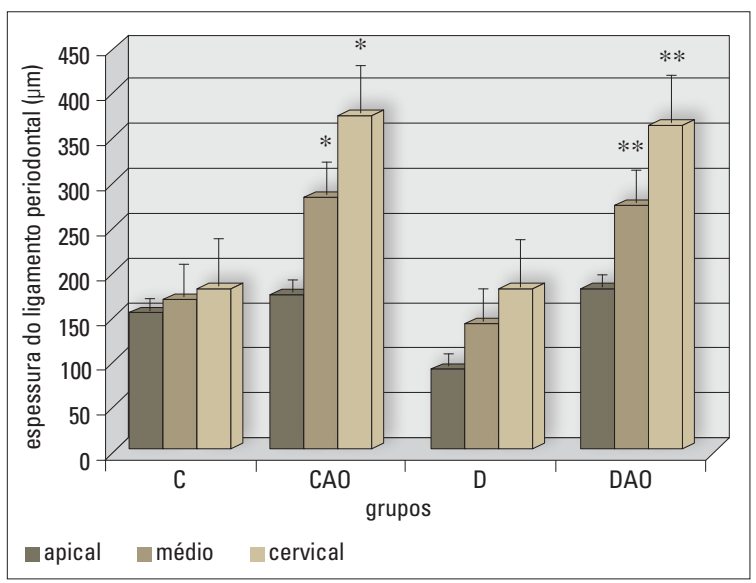

GRÁFICO 2 - Espessura do ligamento periodontal ( $\mu \mathrm{m})$ nos terços apical, médio e cervical da raiz observada nos grupos: $\mathrm{C}$ - animais normoglicêmicos não submetidos a forças ortodônticas; CAO - animais normoglicêmicos submetidos a forças ortodônticas; $D$ - animais diabéticos não submetidos a forças ortodônticas; DAO - animais diabéticos submetidos a forças ortodônticas $(\mathrm{n}=5$ ratos por grupo; ${ }^{*} \mathrm{p}<0,05$ quando comparado ao grupo $\mathrm{C} ;{ }^{* *} \mathrm{p}<0,05$ quando comparado ao grupo $D)$. 

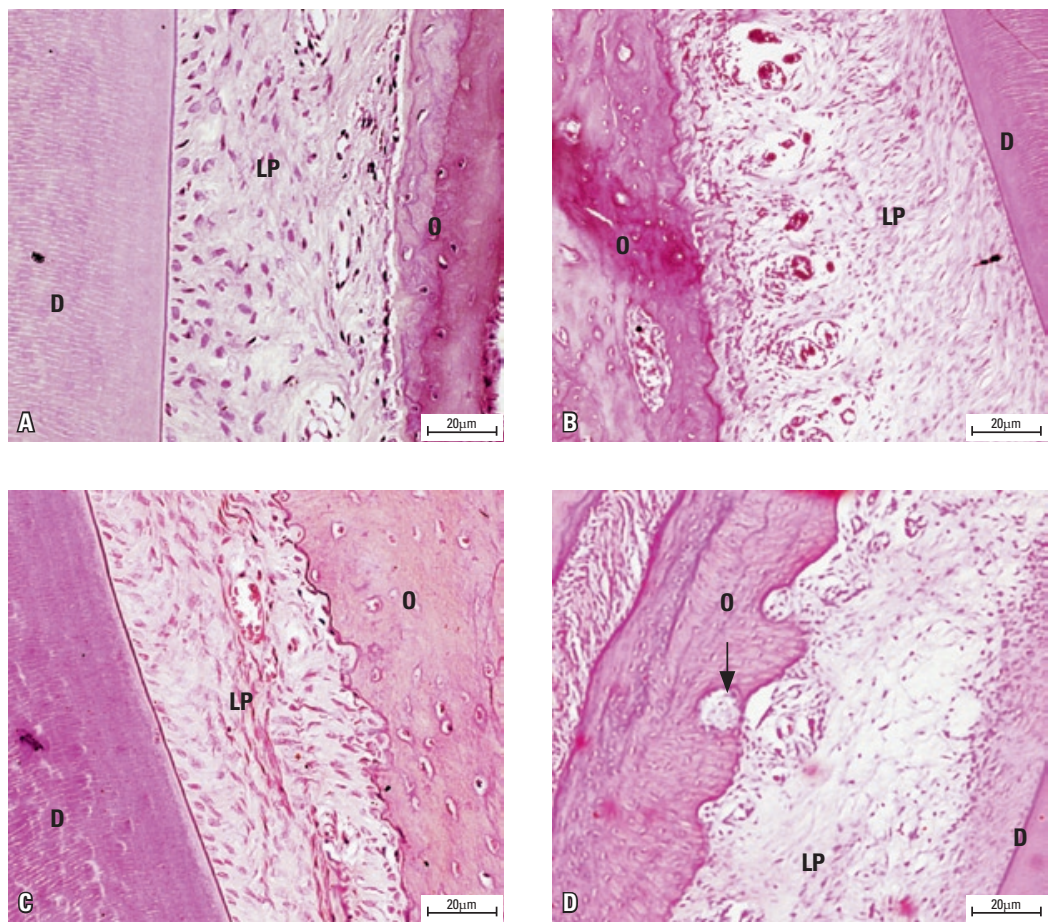

FIGURA 2 - Fotomicrografia evidenciando o periodonto de ratos pertencentes aos grupos: C - animais normoglicêmicos não submetidos a forças ortodônticas (A); CAO - animal normoglicêmicos submetidos a forças ortodônticas (B); D - animais diabéticos não submetidos a forças ortodônticas (C); DAO - animais diabéticos submetidos a forças ortodônticas (D) ( $\mathbf{L P}=$ ligamento periodontal, seta $=\mathbf{B M U}, \mathbf{D}=$ dentina radicular, $\mathbf{0}=0$ osso alveolar).

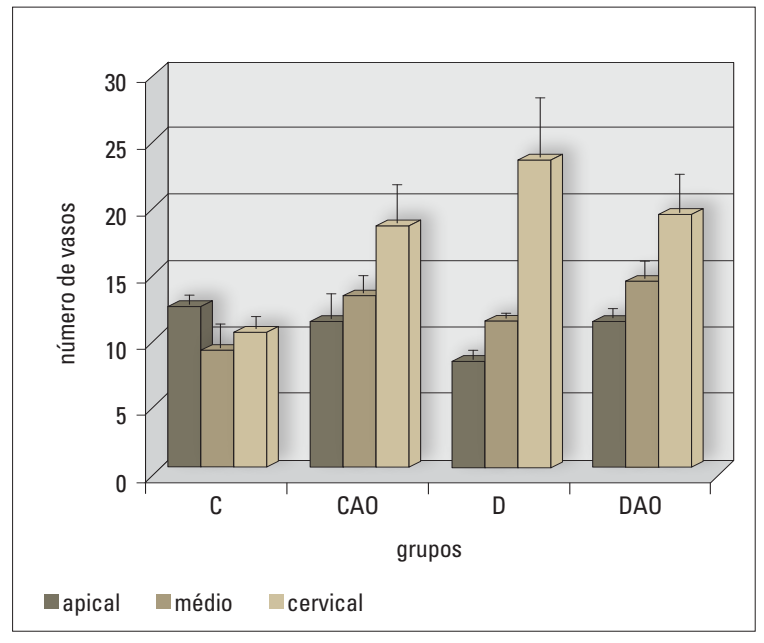

GRÁFICO 3 - Número de vasos sanguíneos nos terços apical, médio e cervical da raiz, observado nos grupos: $\mathrm{C}$ - animais normoglicêmicos não submetidos a forças ortodônticas; CAO - animais normoglicêmicos submetidos a forças ortodônticas; D - animais diabéticos não submetidos a forças ortodônticas; DAO - animais diabéticos submetidos a forças ortodônticas ( $n=5$ ratos por grupo, $p>0,05$ quando todos os grupos foram comparados).

\section{DISCUSSÃO}

O modelo de diabetes experimental mostrouse eficiente, pois em todos os animais dos grupos $\mathrm{D}$ e DAO observou-se a presença de polifagia, poliúria e polidpsia e também um menor peso corporal, quando comparado aos animais normoglicêmicos (grupos $\mathrm{C}$ e CAO).

O peso dos animais do grupo controle não variou, enquanto os animais do grupo CAO emagreceram uma quantidade significativa para somente 5 dias de experimento. Esse menor peso evidenciado nos animais do grupo CAO pode ser devido ao dispositivo ortodôntico e também devido à presença das aftas, o que provavelmente incomodou os animais ao se alimentarem ${ }^{1}$. Os animais do grupo D e DAO também sofreram uma redução de peso. No grupo $\mathrm{D}$, a redução de peso 
foi conseqüência do quadro clínico do diabetes melito e a do grupo DAO foi ainda mais intensificada, pois, além da patologia, o dispositivo de movimentação dentária atrapalhou a alimentação. Nos diabéticos ocorre um distúrbio da síntese e armazenamento de reservas energéticas, em conseqüência da ausência de insulina, acarretando a perda de peso $^{7}$. Nos animais do grupo CAO, mesmo apresentando dificuldade para se alimentarem, os efeitos da perda de peso foram menores que o efeito metabólico causado pelo diabetes melito em apenas 5 dias.

Um outro dado que nos assegura que os animais do grupo D e DAO estavam com diabetes melito é que estes estavam severamente hiperglicêmicos. Mas observou-se que os animais que sofreram movimentação dentária (grupo DAO) apresentavam uma glicemia aproximadamente $30 \%$ menor que os animais do grupo D. Esse fato está relacionado à dificuldade que esses animais apresentaram para se alimentar, com conseqüente menor ingestão de alimento.

Baseado em estudos prévios em ratos ${ }^{1,11,13}$, elegeu-se o período de 5 dias de movimentação dentária induzida e uma força de $20 \mathrm{~g}$. A escolha se restringiu somente a animais machos, para se evitar eventuais variações hormonais que são características do gênero feminino. Ao contrário do relatado por Akin et al. ${ }^{1}$, que consideraram o dispositivo ortodôntico inócuo ao tecido mole, no presente estudo foi observada - em todos os animais dos grupos CAO e DAO - a presença de lesões de tecido mole, na região da comissura labial, a partir de 2 dias da colocação dos dispositivos de movimentação dentária. Embora no início da movimentação se apresentassem afastados do tecido mole, após a sua atuação, aproximaram-se das mucosas. Num futuro estudo que, por ventura, utilize esse método, pode-se evitar isso mediante a colocação de uma proteção, por exemplo, de resina composta, nas extremidades do fio de aço.

A medição da distância interincisivos deixou evidente que a movimentação ortodôntica não diferiu entre os ratos controles e os diabéticos, sendo a distância praticamente idêntica nos grupos CAO e DAO. Dois animais do grupo DAO apresentaram um dente com aparência de anquilose, pois estavam menos irrompidos que seus adjacentes, entretanto histologicamente não apresentaram nenhuma anormalidade. Esse fato pode ser decorrente de uma alteração no ritmo de irrupção entre estes dentes, já que nos ratos os incisivos apresentam irrupção contínua.

$\mathrm{O}$ número de vasos sanguíneos encontrados no ligamento periodontal não diferiu estatisticamente quando foram comparados os terços apical, médio e cervical dos quatro grupos estudados. Porém, observou-se a presença de um número maior de vasos sanguíneos no terço cervical nos grupos CAO, D e DAO, sendo em média o dobro do encontrado no grupo C. Houve microangiopatias nos animais dos grupos D e DAO, com aparência de quadros hemorrágicos em grandes áreas e em várias partes do ligamento periodontal. Holtgrave et al. ${ }^{9}$ estudaram reações no periodonto em conseqüência de forças ortodônticas e observaram a presença de microangiopatias na gengiva, concluindo que mudanças específicas no periodonto são mais pronunciadas depois do movimento ortodôntico. As microangiopatias são observadas em pacientes diabéticos, inclusive os que apresentam os níveis glicêmicos controlados ${ }^{2}$. Essas alterações vasculares ocorrem, provavelmente, devido ao enfraquecimento da parede dos vasos em decorrência do diabetes melito, que atua interferindo na sintese do colágeno ${ }^{14}$.

As médias da espessura do ligamento periodontal nas três regiões analisadas para cada grupo estudado foram, nos grupos C, CAO, D e DAO, respectivamente, de $163,3 \mu \mathrm{m}, 273,3 \mu \mathrm{m}, 136 \mu \mathrm{m}$ e $270 \mu \mathrm{m}$. Possivelmente, a maior espessura do ligamento periodontal dos grupos $\mathrm{CAO}$ e DAO, em relação aos grupos controles (C e D), como esperado para o lado de tensão, foi marcada pelo estiramento das fibras colágenas decorrente da movimentação ${ }^{4}$. 
Numa análise qualitativa da face óssea, foi observada a presença de uma grande quantidade de lacunas de reabsorção por toda a superfície óssea, desde a região apical até a cervical, principalmente nos cortes histológicos dos animais diabéticos (grupo D e DAO). Os processos de reabsorção no tecido ósseo dos animais diabéticos (grupo D) sem movimentação ortodôntica ocorrem da mesma maneira que nos animais do grupo $\mathrm{DAO}$, pois, em conseqüência do diabetes melito, o peptídeo amilina está ausente. Este peptídeo é co-secretado pelas células beta do pâncreas e, em condições normais, se liga a receptores para calcitonina, reduzindo os níveis plasmáticos de cálcio. Essa redução inibe a atividade dos osteoclastos e estimula os osteoblastos ${ }^{10}$. Devido ao fato da amilina estar ausente nos diabéticos, os processos de reabsorção óssea são ativados, pois os osteoclastos deixam se ser inibidos. Nos animais do grupo $\mathrm{DAO}$, a reabsorção óssea ocorre em conseqüência da patologia do diabetes melito e também devido à movimentação dentária. Mesmo no lado de tensão, as células se tornam deformadas e, em hipóxia, liberam proteínas livres e mediadores químicos responsáveis pela reabsorção óssea ${ }^{4}$.

Torna-se oportuno ressaltar que todos os modelos de supressão total de um componente orgânico nutricional ou hormonal, como o presente estudo que simulou o diabetes melito não controlado, devem ser interpretados com cautela, já que não reproduzem totalmente a realidade clínica ${ }^{4}$. Os pacientes ortodônticos apresentamse, na maioria das vezes, controlados. Esse fato nos conduz a uma futura pesquisa, na qual os ratos diabéticos receberão insulina em doses diferentes para reproduzir os pacientes controlados e parcialmente controlados. Dessa forma, aproximando-se mais das condições em que os pacientes apresentam-se para o tratamento ortodôntico.

Mesmo diante dessa observação, a possivel perda óssea marginal, corroborada no presente trabalho, deve ser motivo de preocupação, já que Bensch et al. ${ }^{2}$ relataram que as angiopatias podem ocorrer mesmo em diabéticos com níveis glicêmicos corrigidos.

\section{CONCLUSÕES}

As modificações no lado de tensão do ligamento periodontal de ratos diabéticos, diante da movimentação dentária induzida, são mais pronunciadas dos que as observadas em ratos normais, bem como apresentam ocorrência de angiopatias e áreas de reabsorção mais evidentes do que os diabéticos sem movimentação dentária, principalmente na região cervical. Essas ocorrências podem favorecer a perda óssea marginal, mesmo no lado de tensão, diante da movimentação dentária induzida. 


\title{
Periodontal ligament changes after induced dental movement in diabetic rats
}

\begin{abstract}
Aim: The aim of this study was to evaluate the periodontal ligament changes after induced dental movement of the upper incisor in diabetic rats. Methods: Twenty Wistar rats (Rattus norvegicus) with 105 days of age were used. The rats were divided in four groups: C - normoglicemic animals not submitted to dental movement; CAO normoglicemic animals submitted to dental movement; D - diabetic animals not submitted the dental movement; DAO - diabetic animals submitted to dental movement. The animals had remained with dental movement devices during 5 days. The number of sanguine vessels and the thickness of the periodontal ligament were evaluated at cervical, medium and apical histological cut regions. Results and Conclusion: At tension side, the dental movement in the animals of group CAO resulted in a thicker periodontal ligament (17.64\% apical, 39.28\% medium, 51.35\% cervical) when compared to $C$ group ( $p<0.05$ for medium and cervical area). Group DAO exhibited an increase of periodontal ligament thickness of $50.55 \%$ (apical), $48.14 \%$ (average) and $50 \%$ (cervical) when compared to group $D(p<0.05)$. The periodontal ligament sanguine vessels number did not differed significantly for all groups $(p<$ 0.05). At tension side, bone reabsorption lacunae were observed in CAO, D and DAO groups. The pressure side was not examined in this study phase.
\end{abstract}

Key words: Dental movement. Diabetes mellitus. Periodontal ligament.

\section{REFERÊNCIAS}

1. AKIN, E. et al. Effects of nitric oxide in orthodontic tooth movement in rats. Am. J. Orthod. Dentofacial Orthop., St. Louis, v. 126, no. 5, p. 608-614, 2004.

2. BENSCH, L. et al. Orthodontic treatment considerations in patients with diabetes mellitus. Am. J. Orthod. Dentofacial Orthop. St. Louis, v. 123, no. 1, p. 74-78, 2003.

3. BERGMEYER, H. U. et al. Determination of glucose with glucose oxidase and peroxidase: methods of enzymatic analysis. New York: Verlag Chemiem-Academic, 1974.

4. CONSOLARO, A. Reabsorções dentárias na especialidade clínica. 2. ed. Maringá: Dental Press, 2005.

5. DUARTE, C. A. et al. Alterações periodontais em pacientes diabéticos. Odontol. Paulista, São Paulo, v. 2, p. 11-14, 1984.

6. FINNEY, L. S. What the mouth has to say about diabetes. Postgrad Med., Bombay, v. 102, p. 117-126. 1997.

7. GUYTON, A. C. Tratado de fisiologia médica. 10. ed. Rio de Janeiro: Guanabara Koogan, 2002.

8. HILL, P. A. et al. Bone remodeling. Br. J. Orthod., London, v. 25 , no. 2 , p. 101-107, 1998.

9. HOLTGRAVE, E. A. et al. Periodontal reactions to orthodontic forces in the diabetic metabolic state. Forstschr Kieferorthop., München, v. 50, no. 4, p. 326-337, 1989
10. HORCAJADA-MOLTENI, M. N.: CHANTERANNE, B.: LEBECQUE, P.; DAVICCO, M. J.; COXAM, V.; YOUNG, A.; BARLET, J. P. Amylin and bone metabolism in StreptozotocinInduced diabetic rats. J. Bone Miner. Res., Washington, DC, v. 16 , no. 5, p. 958-965, May 2001.

11. KVINNSLAND, S. et al. Effect of experimental tooth movement on periodontal and pulp blood flow. Eur. J. Orthod., London, v. 11, p. $200-205,1989$.

12. LOE, H. Periodontal disease: the sixth complication of diabetes mellitus. Diabetes Care, New York, v. 16, p. 329-334, 1993.

13. REN, Y. et al. Optimum force magnitude for orthodontic tooth movement: a systematic literature review. Angle Orthod., Appleton, v. 73, no. 1, p. 86-92, 2003.

14. SAODOUN, A. Diabetes and periodontal disease: a review and update. J. West Soc. Periodontol. Periodontal, Santa Monica, v. 28, p. 116-139, 1980.

15. SILVERTHORN, D. U. et al. Fisiologia humana: uma abordagem integrada. Barueri: Manole, 2003. p. 816.
Endereço para correspondência

Luis Alberto Sabino Vila Real

Avenida Brasil, 1120, sala 2 - Centro

CEP: 86.870-000 - Ivaiporã/PR

E-mail: luis_vilareal@yahoo.com.br 